\title{
Avant-propos
}

\section{F. Pourrat}

Spécialiste qualifié en ODF, Pratique privée

Pourquoi ce numéro de la Revue d'ODF ? Pourquoi ce thème ? Pourquoi ces auteurs?

Au siècle dernier, en 1983, avec des amis dentistes, nous fondions une association, la SPDO, la Société pluridisciplinaire d'odontologie dont le but était, par des passionnés et pour des passionnés, d'échanger leurs compétences dans tous les domaines de la dentisterie afin d'optimiser la réalisation des traitements pluridisciplinaires. Chacun de nous était dépourvu d'arguments face au manque de compréhension de l'autre, au manque de savoir et de compétences dans les différents domaines.

Aujourd'hui, en 2015, est née une association de jeunes odontologistes passionnés qui eux aussi veulent apprendre, comprendre et partager leur savoir et leurs compétences dans les différentes spécialités odontologiques.

Ils se rassemblent sous I'association Friendly Study Club, initiée par Gary Finelle, Marie Medio et Antoine Popelut.

Il nous a semblé opportun de leur demander de mettre à niveau les connaissances dans les différents domaines autour de l'orthodontie, pour aider les orthodontistes à pouvoir répondre au questionnement des patients et aussi à appréhender les exigences des odontologistes pour assurer les traitements pluridisciplinaires: meilleure compréhension du problème, meilleure planification, meilleure communication, meilleure satisfaction du patient.

Le thème sera donc " autour de l'orthodontie » et bien sûr, nous ne pourrons pas envisager la totalité des savoirs des spécialités à nos côtés qui sont aujourd'hui trop vastes.

Julien Philippe nous conforte, par son éditorial, dans la réalité de l'orthodontie, à la fois proposition d'une orthodontie médicale consécutive à un diagnostic de la maladie ou de la dysfonction et d'une orthodontie correctrice des difformités. II nous réitère l'importance de notre rôle pour améliorer la fonction et la forme.

Puis, au cours de ce numéro résolument axé vers les autres spécialités de l'odontologie, les auteurs ne donnent qu'une infime petite partie de l'état actuel des connaissances mais ô combien importante pour nous.

Anthony Atlan nous éclaire sur le diagnostic et la prise en charge des taches de l'émail. Gary Finelle nous 
détaille ensuite la prévisualisation du projet thérapeutique tel que le permettent les outils numériques actuels. Dans la réalité des traitements pluridisciplinaires, il est bien de reconnaître leurs bienfaits. Pour atteindre les objectifs de manière plus sereine, Frank Pourrat aborde un outil managérial de gestion de projet orienté odontologie. Antoine Popelut nous fait comprendre que la gestion des tissus mous est majeure pour la réussite des thérapeutiques parodontales. Maxime Drossart fait la démonstration de l'intérêt actuel des bridges cantilever et Sylvain Mouraret insiste sur la nécessité de prendre en charge l'attache parodontale avant le traitement orthodontique. Nous ne pouvions pas, Marie Medio et moi, rendre compte des aides techniques sans parler de la temporisation des dents absentes par l'apport des mini-vis.

La pluridisciplinarité est bien là. Le traitement peut se finaliser et vous découvrirez dans l'article de Marie
Medio l'apport de la CFAO dans le maintien de nos traitements.

Pour les passionnés d'orthodontie, un cas clinique présenté par Patrick Guezenec.

II manque un certain nombre de pages pour parler d'autres spécialités comme l'orthophonie, l'implantologie, la chirurgie maxillo-faciale, la diététique, la psychologie, etc. Mais ce groupe du Friendly Study Club a réalisé un magnifique travail et je tiens à les remercier de nous apporter autant de savoirs actualisés.

L'intelligence collective est nécessaire aussi dans la pluridisciplinarité.

Anne Nelly Perret-Clermont (2003) la définit comme " I'art de maximaliser simultanément la liberté créatrice et l'efficacité collaborative ».
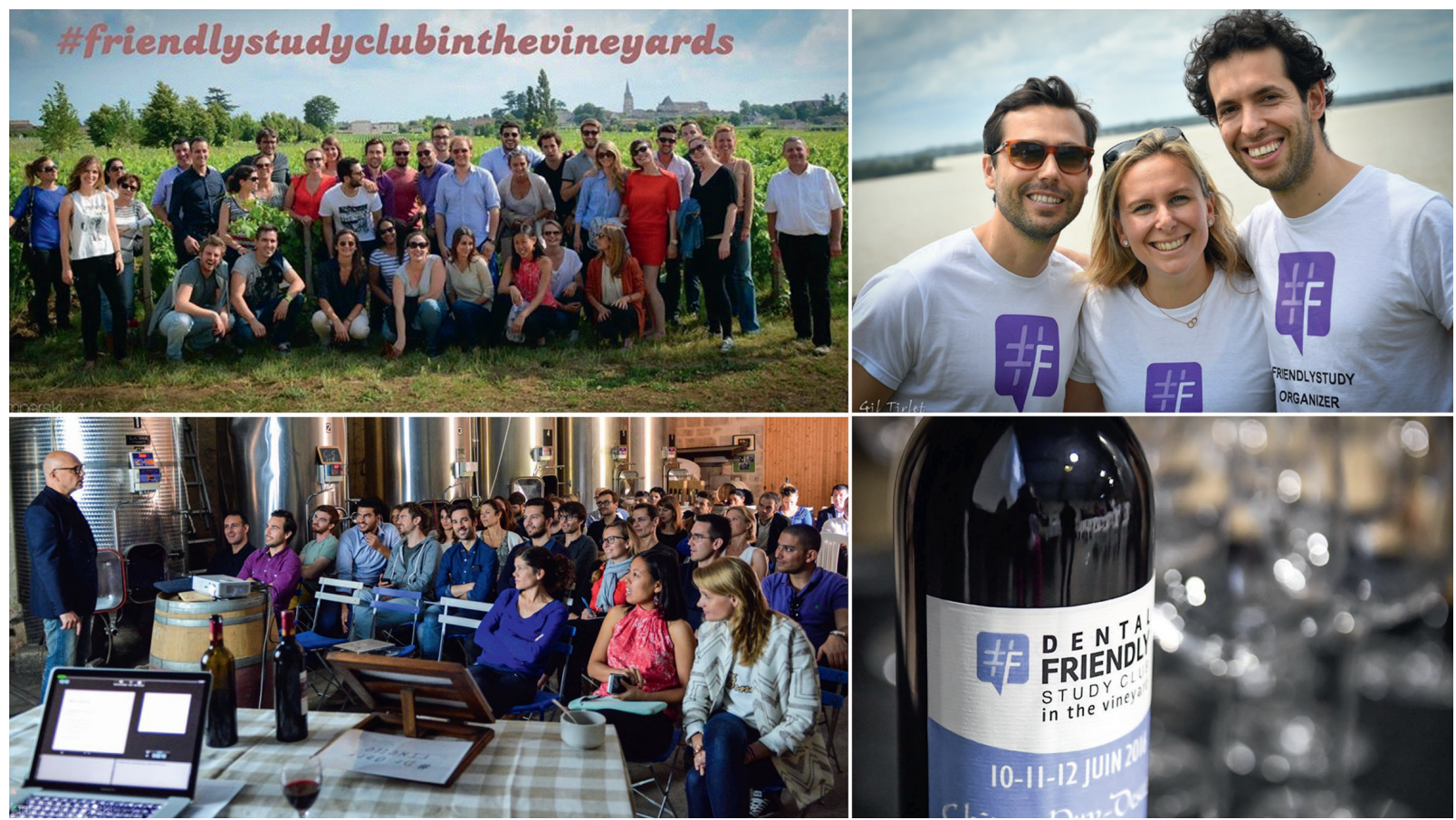

"A vaincre sans péril, on triomphe sans gloire »

Pierre Corneille, Cid, Acte II, Scène 2 